\title{
Laboratórios educacionais virtuais como promotores do estado de flow e da aprendizagem ativa.
}

\author{
Leandro Rosniak Tibola - PPGIE/UFRGS (1rtibola@gmail.com) \\ Liane M. Rockenbach Tarouco - PPGIE/UFRGS (liane@penta.ufrgs.br)
}

Resumo. As novas gerações de alunos que estão nas salas de aula são habituadas com os jogos eletrônicos e com a permanente conexão com a Internet. Frente a esta nova realidade, a educação enfrenta o desafio de utilizar recursos tecnológicos semelhantes aos que o aluno está acostumado, proporcionando atividades práticas em laboratório; de forma que os conceitos teóricos recebidos em sala de aula possam ser aplicados em um ambiente virtual gamificado. Este trabalho apresenta um Laboratório Educacional Virtual (LEV) que busca propiciar o estado de Flow e a aprendizagem ativa. O LEV foi validado por 49 estudantes, dos 15 aos 20 anos, os quais apresentaram indícios de Flow e melhoria na aprendizagem.

Palavras-chave: Laboratório Educacional Virtual. Estado de Flow. Aprendizagem Ativa. Gamificação. Mundos Virtuais.

\section{VIRTUAL EDUCATIONAL LABS AS PROMOTERS OF THE FLOW STATE AND THE ACTIVE LEARNING}

Abstract. The new generations of students in classrooms are accustomed to electronic games and the permanent connection to the Internet. Faced with this new reality, education confront the challenge of using technological resources similar to those that the student is accustomed to, providing practical activities in the laboratory; so that theoretical concepts received in the classroom can be applied in a virtual environment. This work presents a Virtual Educational Laboratory (VEL) that seeks to provide the state of Flow and active learning. The VEL was validated by 49 students, from 15 to 20 years old, who presented signs of Flow and improvement in learning.

Keywords: Virtual Educational Lab. Flow State. Active Learning. Gamification. Virtual Worlds.

\section{Introdução}

Nos últimos anos, tem-se observado a intensidade com que os alunos usam os recursos tecnológicos, principalmente a Internet e os jogos digitais (CETIC.BR, 2017). Porém, cabe apontar que, apesar destas ferramentas serem bastante familiares aos alunos, elas são oportunidades ainda pouco exploradas na educação (NMC e FIRJAN, 2012). Estas preocupações são ainda mais pertinentes, uma vez que a pesquisa "TIC Educação 2016" (CETIC.BR, 2017, pág. 99), indica uma tendência de redução no uso dos laboratórios de informática para atividades relacionadas com a Internet, o que diminui ainda mais a possibilidade de uso dos laboratórios virtuais. Estas preocupações, somadas a apreensão em torno do equilíbrio entre o domínio teórico e prático na formação acadêmica, como levantadas por Feisel e Rosa (2005) e Auer et al. (2003), possibilitaram identificar um campo de pesquisa à ser explorado.

Neste contexto, considerando a necessidade de atividades práticas em várias disciplinas; o alto custo envolvido com os laboratórios educacionais; a indisponibilidade e, mais recentemente, a redução do uso deste tipo de laboratórios nas escolas; a frequência com que os alunos possuem recursos tecnológicos mais atualizados e atrativos que os disponibilizados pelas escolas; surge a questão norteadora desta pesquisa: "a construção de laboratórios educacionais em mundos virtuais pode 
apresentar potencial para engajar o aluno tão profundamente a ponto de levá-lo ao estado Flow e assim, pode haver melhor desempenho nas atividades acadêmicas?".

O presente trabalho está assim organizado: a seção 2 relata brevemente os fundamentos teóricos que embasam este trabalho; a seção 3 apresenta a estrutura da pesquisa e descreve brevemente o LEV; seguida pela seção 4, com as análises dos dados coletados; a seção 5, com a discussão dos resultados decorrentes da pesquisa e por fim, na seção 6 são descritas as conclusões e os trabalhos futuros.

\section{Fundamentação Teórica}

\subsection{Laboratórios de práticas}

Considerando as exigências da Lei de Diretrizes e Bases da Educação Nacional (LDB) em relação aos laboratórios (BRASIL, 1996) e a necessidade do fortalecimento da relação teórico-prática nas salas de aula, é necessário identificar os tipos e as classes dos laboratórios. Os laboratórios podem ser de três tipos: (a) de pesquisa, quando geram a base de conhecimento para outras pesquisas que vão aplicá-la; (b) de desenvolvimento, que são usados para criação de produtos e (3) de educação, os quais objetivam consolidar nos estudantes o conhecimento teórico através da prática (FEISEL e ROSA, 2005). Já em relação à classificação, Auer et al. (2003) classificam os laboratórios de acordo com a localização dos estudantes e do tipo de experimento: (I) laboratórios tradicionais, (II) laboratórios remotos, (III) simulação local e (IV) laboratório virtual. Então, buscando uma nomenclatura que possa representar melhor o laboratório tratado neste trabalho, agrupou-se a tipologia de Feisel e Rosa (2005) com a classificação de Auer et al. (2003) obtendo a denominação "laboratório educacional virtual" ou LEV, como chamado de agora em diante.

Então, o laboratório educacional virtual é uma alternativa que contorna as questões de custo e segurança dos laboratórios físicos necessários nas escolas e universidades. Os laboratórios educacionais virtuais apresentam algumas vantagens em relação aos laboratórios físicos: (1) seu custo é aceitável; (2) não apresentam risco à saúde do aluno; (3) permitem um grande número de acessos simultâneos nos mesmos períodos; (4) o aluno não precisa estar ou ir até a escola para fazer uma experiência, pode fazê-la de casa ou em trânsito; (5) o aluno pode repetir o experimento.

\subsection{Aprendizagem Ativa}

Além da estrutura e dos suprimentos, para que a aprendizagem em laboratório aconteça, é preciso que o aluno mude sua postura: de apenas observar a experiência para realizá-la, analisá-la e propor novas alternativas para sua realização. Esta mudança de postura se alinha com o que Piaget (1972) chamou de abstração reflexiva, que é o processo pelo qual as propriedades essenciais do objeto são compreendidas, permitindo inferir possíveis reações em outros contextos.

Neste contexto, este trabalho baseou-se na Aprendizagem Experiencial, definida como "o processo pelo qual o conhecimento é criado através da transformação da experiência, o conhecimento resulta da combinação de compreender e transformar a experiência" (KOLB ,1984, p. 41). A Aprendizagem Experiencial retrata dois modos de compreender a experiência: Experiência Concreta (EC) e Conceituação Abstrata (CA), e dois modos de transformar a experiência: Observação Reflexiva (OR) e Experimentação Ativa (EA). De acordo com o ciclo de aprendizagem em quatro fases, as experiências concretas ou imediatas são a base para observações e reflexões. Estas reflexões são assimiladas e refinadas em conceitos abstratos a partir do qual novas implicações para a ação podem ser extraídas. Estas implicações podem ser ativamente testadas e servem como guia para a criação de novas experiências (KOLB, 1984). 


\subsection{Estado de Flow}

Flow é um estado subjetivo que as pessoas relatam quando elas estão completamente envolvidas em algo a ponto de esquecer o tempo, fadiga e tudo o mais, exceto a própria atividade. A característica determinante de Flow é o intenso envolvimento experiencial em uma atividade, a cada momento de sua realização. A atenção está totalmente voltada para tarefa a ser realizada e as pessoas funcionam em sua plena capacidade (CSIKSZENTMIHALYI, 1990).

Assim, para que o estado de Flow ocorra, três condições chaves são necessárias: (I) a atividade deve apresentar um conjunto claro de objetivos; (II) existência de equilíbrio entre desafios percebidos e habilidades dominadas; e (III) feedback claro e imediato. Sob estas condições, o indivíduo entra em um estado subjetivo com as seguintes características: (1) concentração intensa na atividade sendo executada; (2) fusão da ação e da consciência; (3) perda da autoconsciência reflexiva; (4) sensação de controle; (5) distorção da experiência temporal; (6) sentimento da atividade como intrinsecamente gratificante (CSIKSZENTMIHALYI, 1990).

Quando está em estado de Flow, o indivíduo opera com sua capacidade total. Mas, o estado de Flow é um estado de equilíbrio dinâmico: depende do estabelecimento de um equilíbrio entre as capacidades de ação percebidas e as oportunidades de ação disponíveis. O equilíbrio é intrinsicamente volátil. As mudanças no estado subjetivo fornecem o feedback sobre a mudança de relacionamento do indivíduo com o ambiente. Vivenciar ansiedade ou tédio pressiona o indivíduo para ajustar o seu nível de habilidade e/ou desafio, a fim de escapar do estado aversivo e retornar ao estado de Flow (CSIKSZENTMIHALYI, 1990).

\subsection{Mundos Virtuais Digitais 3D}

Os mundos virtuais são mundos em 3D gerados por computador, que permitem a representação do mundo físico de forma realista (NELSON e ERLANDSON, 2012). Quando um usuário acessa o mundo virtual, ele é representado naquele ambiente por um avatar, que é considerado como um cibercorpo inteiramente digital, uma figura gráfica de complexidade variada que empresta sua vida simulada para o transporte de cibernautas para dentro dos mundos do ciberespaço. A transposição para o mundo virtual, a estrutura em sua volta, a interação com outros avatares e com os objetos presentes no ambiente fornece ao indivíduo uma forte sensação de presença no mundo digital (SANTAELLA, 2003). Os mundos virtuais apresentam características que podem possibilitar condições criar o estado de Flow e auxiliar na aprendizagem, são elas: imersão (o usuário se sente "dentro" do mundo virtual, faz parte do cenário), realismo (capacidade dos ambientes digitais representarem o mundo físico no mundo virtual com grande semelhança), e interação (a ação desencadeada pelo avatar provocará a reação de um objeto tridimensional).

\subsection{Gamificação}

A gamificação é uma estratégia na qual se aplicam técnicas de design de jogos para contextos de não-jogos, a fim de dirigir o comportamento do usuário (GAMIFICATION.ORG, 2010). Outros autores consideram a gamificação no seu aspecto estrutural, tal como Busarello; Ulbricht e Fadel (2014), para quem a gamificação tem como base a ação de se pensar como em um jogo, utilizando as sistemáticas e mecânicas do ato de jogar em um contexto fora de jogo. Já para Kapp (2012), a gamificação prevê a utilização de mecânicas, estéticas e características dos jogos para envolver e motivar as pessoas para aprender e para resolver problemas. Ainda para Schäfer e Lopes (2011), a gamificação pode ser definida como a aplicação da lógica e da mecânica dos games em diferentes aspectos do cotidiano, caracterizando, 
portanto, ambientes que contenham elementos de jogos como gamificados. Pode-se entender o método de gamificação como o uso de elementos do design dos jogos para alcançar objetivos em comum. Devido à abordagem deste artigo, as técnicas de gamificação utilizadas na pesquisa não serão descritas.

\subsection{Trabalhos correlatos}

Como forma de situar esta pesquisa em relação ao estado da arte, os seguintes trabalhos, desenvolvidos no meio acadêmico e de livre acesso/distribuição, foram estudados: Techland (OCCHIONI, 2013), SciEthics Interactive (SCIETHICS INTERACTIVE, 2013) e EcoMUVE (HARVARD, 2011). Ainda, foram avaliados em seus aspectos semelhantes aos deste trabalho o Labster (LABSTER, 2018) e o CloudLabs (E-TECH, 2017), porém os mesmos são produtos comerciais. Devido ao tamanho do texto, maiores informações podem ser obtidas nas referências dos mesmos.

\section{Configuração da Experiência}

O Laboratório Educacional Virtual, no qual foi desenvolvida a presente pesquisa, é um mundo virtual que aborda conteúdos de Eletricidade e disponibiliza: (1) materiais instrucionais - vídeos, páginas Web e apresentações; (2) experimentos - conjunto de objetos 3D que realizam experiências relacionadas aos conteúdos; e (3) questionários abordando os sobre os assuntos tratados. Os alunos podem interagir com todos os componentes do mundo virtual, em qualquer ordem e quantas vezes desejar.

O LEV foi avaliado por 49 alunos (36 alunos do ensino médio e 14 alunos do $2^{\circ}$ semestre de Ciência da Computação) de uma Universidade do Rio Grande do Sul. Destes alunos, 27 eram homens e 22 eram mulheres e as idades variaram dos 15 aos 20 anos, com média de 16,4 anos e desvio padrão de 7,4. A escolha destes alunos de deve ao fato dos professores das disciplinas de Física serem os mesmos, facilitando o dimensionamento dos conteúdos nos laboratórios e avaliação posterior. Antes da experiência, os alunos foram informados dos objetivos e procedimentos da pesquisa e o caráter estritamente voluntário da mesma. Os alunos menores de idade foram devidamente autorizados pelos pais, todos os alunos preencheram os documentos legais necessários e a pesquisa foi aprovada pelo Comitê de Ética em Pesquisa. A avaliação compreendeu, inicialmente, de uma breve explanação sobre o mundo virtual e sobre os procedimentos na experiência; o preenchimento de um formulário para identificação do perfil de domínio tecnológico do aluno; e a realização do pré-teste. Então, os alunos utilizaram o LEV. Após a utilização do laboratório educacional virtual, os alunos preencheram o Questionário de Flow, adaptado da Flow State Scale-2 (FSS-2), descrito por Jackson; Eklund e Martin (2010), seguido pelo pós-teste e pelo questionário de avaliação da experiência.

Os dados analisados nesta pesquisa foram obtidos de dois modos, através dos questionários impressos e do registro digital das ações do avatar do aluno no LEV. Os questionários preenchidos pelos alunos compreenderam: (1) Perfil tecnológico do aluno, (2) Pré-teste, (3) Pós-teste, (4) Questionários de Flow e (5) Avaliação do Laboratório Educacional Virtual. Já o registro digital utilizou as tabelas: (I) "apresentacao" armazena todas as interações entre o avatar e os objetos presentes no LEV; (II) "hotpotatoes" - guarda os resultados dos testes eletrônicos (quizzes) respondidos pelos alunos no LEV; (III) "pontos_tarefas"- salva a pontuação obtida pelo aluno ao completar as atividades previstas no LEV; e (IV) "trajeto" - armazena o percurso realizado pelo avatar do aluno tanto no ambiente quanto e no LEV. A detecção da movimentação e das interações do avatar é realizada pelos sensores de localização geográfica e de interação, que são melhores detalhados em Tibola e Tarouco (2015). 


\subsection{Laboratório Educacional Virtual - LEV}

O Laboratório Educacional Virtual dispõem de três estruturas diferentes: a Entrada Principal, a Praça Central e o Prédio das Experiências. A Entrada Principal é o ponto inicial do aluno no mundo virtual, onde estão disponíveis: um vídeo descrevendo os objetivos do LEV, as informações do funcionamento dos materiais instrucionais, os controles do avatar e do mundo virtual 3D. Ainda, é o primeiro contato com a Tutora Atena, um chatterbot conversacional que auxilia o aluno tanto com os conteúdos abordados, quanto com o mundo virtual. Devido ao foco deste artigo, o funcionamento, a estrutura e os conceitos relacionados com a Tutora Atena não serão abordados aqui, mas podem ser encontrados no Projeto AVATAR (http://www.ufrgs.br/avatar). A Figura 1-A mostra parcialmente a Entrada Principal. Após tocar no teletransporte, o aluno é levado para a Praça Central, onde o aluno acessa informações sobre a estrutura dos prédios e a forma de utilização dos laboratórios do Prédio das Experiências.

Chegando ao Prédio das Experiências, o aluno pode preencher um questionário para verificação do seu nível de conhecimento. Seguindo adiante, o aluno terá acesso ao material instrucional, na forma de texto e imagens, páginas Web, vídeos $\mathrm{e}$ apresentações. No final de cada espaço o aluno pode preencher o questionário do respectivo conteúdo. A Figura 1-B apresenta um local com material instrucional.

Após examinar o conteúdo proposto, o aluno alcança a Sala de Eletrização, onde estão disponíveis os experimentos de atração e repulsão do átomo, sentido da corrente elétrica, eletrização em materiais isolantes e eletrização em materiais condutores. Ao chegar ao $2^{\circ}$ andar, o aluno pode fazer uma revisão dos conteúdos apresentados anteriormente. $\mathrm{Na}$ sequência estão disponíveis a apresentação, o experimento e o questionário eletrônico sobre "Eletrização, condutores e aterramento", sendo este experimento apresentado na Figura 1-C. Indo adiante, o aluno visualiza a apresentação "Eletrização por contato e por indução" e pode praticar as respectivas teorias nos laboratórios de "Eletrização por contato" e "Eletrização por indução", mostrados na Figura 1-D e na Figura 1-E respectivamente.

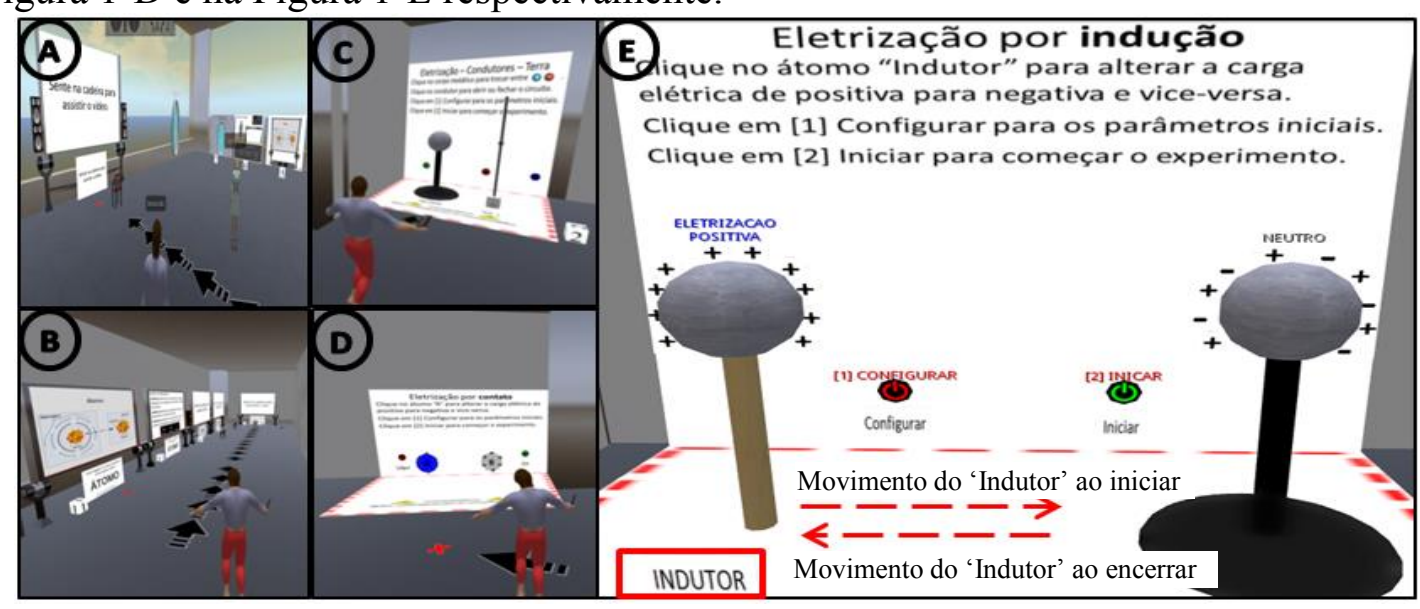

Figura 1 - Ambientes do Laboratório Educacional Virtual.

Mais detalhadamente, para iniciar o experimento de "Eletrização por indução", o aluno deve clicar no botão 'Configurar', para que os objetos do experimento sejam corretamente posicionados. Então, ao clicar no objeto 'Indutor', a eletrização positiva pode ser trocada para negativa e vice-versa. Ao pressionar o botão 'Iniciar', o aluno pode observar o objeto 'Indutor' se aproximar do objeto 'Induzido', alterando no 'Induzido' a disposição dos elétrons contrários aos da carga do 'Indutor', aproximando estes elétrons do objeto 'Indutor'. Ao final do experimento, os objetos voltam à condição inicial e ficam disponíveis para novas experiências. 
Por fim, o aluno chega ao $3^{\circ}$ andar, onde está disponível o experimento de "Eletrização, Indução e Aterramento", um questionário eletrônico verificando o conhecimento dos conteúdos visualizados e um painel para verificação do desempenho geral em todos os questionários. Como forma de gamificação, a pontuação obtida nos questionários foi contabilizado para estabelecer um ranking da pontuação, e pode ser visualizado no objeto de verificação de desempenho. $\mathrm{O}$ arquivo do LEV (Laboratório PA04) pode ser baixado de http://www.ufrgs.br/avatar em "Arquivos e manuais".

\section{Análises e resultados}

Uma vez que esta pesquisa buscou identificar o estado de Flow alcançado pelos alunos e se este proporcionou melhora no desempenho acadêmico, foram realizadas comparações do universo pesquisado com o grupo dos alunos que responderam os seis testes eletrônicos propostos no LEV (chamado de GA11 e composto por 11 alunos); e com o grupo de alunos com nota igual ou superior a 8,0 no Pós-Teste (denominado POS8 e formado por 26 alunos). Em ambos os grupos os alunos auto relataram escalas de Flow mais elevadas em comparação com o total de alunos. Os cálculos de validação e confiabilidade interna foram realizados com o PASW Statistics.

\subsection{Analise dos questionários}

Tendo as escalas de Flow tabuladas e calculadas, foi possível diferenciar a representatividade das dimensões de Flow para o universo dos indivíduos, para o GA11 e para o POS8. A média das dimensões de Flow para o universo pesquisado foi de 3,72 pontos, com desvio padrão (DP) de 0,48 e com o coeficiente Alfa de Cronbach de 0,86 . Já para os alunos do GA11 a média das dimensões de Flow foi de 3,88 pontos $(\mathrm{DP}=0,44)$ e o Alfa de Cronbach foi de 0,89 ; e para os alunos do grupo POS8, a média das dimensões de Flow foi 3,85 ( $\mathrm{DP}=0,43)$, com um coeficiente Alfa de Cronbach de 0,84. Observando-se as dimensões de Flow, nos três grupos analisados os alunos perceberam as dimensões "Perda da autoconsciência reflexiva" e "Experiência autotélica" de forma mais intensa em sua vivencia de Flow, na respectiva ordem. Da mesma forma, os alunos relataram que as dimensões "Concentração intensa e focada na tarefa" e "Fusão da ação e da consciência" foram percebidas como menos intensidade na experimentação de Flow, respectivamente. As dimensões de Flow para cada grupo podem ser visualizadas na Figura 2.

Ainda, a Figura 2 demonstra que, apesar de apresentar valores maiores para todas as médias das dimensões de Flow, se comparadas com todos os alunos, o grupo POS8 demonstrou variações em menos dimensões que o grupo GA11. Isto possivelmente ocorreu por que os alunos do POS8 perceberam a necessidade de "Feedback imediato", "Distorção da experiência temporal" e "Objetivos claros" de forma muito semelhante ao total de alunos, porém com intensidades, e consequentemente, ordens diferentes. Por outro lado, o grupo de alunos GA11 apresentou uma maior disparidade na intensidade com que perceberam as dimensões de Flow, em relação a todos os estudantes. Provavelmente, por terem tido maior interesse pelo conteúdo abordado, terem efetivado as atividades e consequentemente respondido a todos os testes eletrônicos no LEV; os alunos do GA11 apresentaram as maiores sensações para as dimensões "Equilíbrio entre desafios e habilidades", com a intensa concentração aumentando o sentido de "Transformação do tempo", a qual foi suportada pelo "Feedback imediato", "Sensação de controle" e "Objetivos claros".

Já em relação ao ganho de aprendizagem, foi possível verificar que existe correlação positiva entre o estado de Flow e o desempenho nos testes eletrônicos e entre o estado de Flow e o resultado no Pós-Teste, e entre o estado de Flow e a diferença entre Pré-Teste e Pós-Teste. Embora estas correlações não sejam fortes em qualquer dos 
casos, as mais altas correlações percebidas foram entre a pontuação alcançada na realização das atividades no LEV e a diferença entre Pré e Pós-Teste, com coeficiente de 0,34; e entre o estado de Flow e o desempenho nos testes eletrônicos, com coeficiente de 0,46. Isto permite interpretar que aqueles que se envolveram mais ativamente nas atividades do laboratório educacional virtual, e, portanto, tem pontuações mais elevadas nas atividades realizadas e nos testes eletrônicos, conseguiram um avanço maior entre o que eles sabiam, tal como avaliado no Pré-Teste, e o que eles passaram a saber, tal como avaliado no Pós-Teste. Na Figura 3 podem ser vistos estes indicadores.

\begin{tabular}{|l|c|l|c|l|l|}
\hline \multicolumn{2}{|c|}{ TODOS } & \multicolumn{2}{c|}{ GA11 } & \multicolumn{2}{c|}{ POS8 } \\
\hline Dimensão de Flow & Escala & Dimensão de Flow & Escala & Dimensão de Flow \\
\hline 7) Perda da autoconsciência reflexiva & 4,17 & 7) Perda da autoconsciência reflexiva & 4,32 & 7 ) Perda da autoconsciência reflexiva & 4,32 \\
\hline 9) Experiência autotélica & 4,10 & 9) Experiência autotélica & 4,27 & 9) Experiência autotélica \\
\hline 6) Sensação de controle & 3,83 & 1) Equilibrio entre desafios e habilidades & 3,93 & 6) Sensação de controle \\
\hline 1) Equilíbrio entre desafios/habilidades & 3,78 & 8) Distorção da experiência temporal & 3,93 & 1) Equilibrio entre desafios e habilidades & 3,91 \\
\hline 8) Distorção da experiência temporal & 3,57 & 4) Feedback imediato & 3,82 & 4) Feedback imediato \\
\hline 3) Objetivos claros & 3,56 & 6) Sensação de controle & 3,82 & 8) Distorção da experiência temporal & 3,68 \\
\hline 4) Feedback claro e imediato & 3,55 & 3) Objetivos claros & 3,75 & 3) Objetivos claros \\
\hline 5) Concentração intensa/focada na tarefa & 3,51 & 5) Concentração intensa/focada na tarefa & 3,64 & 5) Concentração intensa/focada na tarefa & 3,60 \\
\hline 2) Fusão da ação e da consciência & 3,44 & 2) Fusão da ação e da consciência & 3,43 & 2)Fusão da ação e da consciência \\
\hline
\end{tabular}

Figura 2: Dimensões de Flow para todos os estudantes, GA11 e POS8.

Também é possível verificar que, tanto os alunos do GA11, quanto do POS8, apresentaram crescimento na média de acertos do Pré-Teste para o Pós-Teste $(13,4 \%$ e $10,6 \%$, respectivamente). Ainda, os grupos GA11 e POS8 exibem aumento no número de alunos com mais de 9 pontos no Pós-Teste em relação ao Pré-Teste $(75 \%$ e $50 \%$, respectivamente).

\begin{tabular}{|c|c|c|c|c|c|c|c|c|c|}
\hline \multirow[t]{2}{*}{ Indicador X Turmas } & \multicolumn{3}{|c|}{ Geral (49) } & \multicolumn{3}{|c|}{ GA11 (11) } & \multicolumn{3}{|c|}{ POS8 (26) } \\
\hline & Pré & Pós & Dif. Pós $\rightarrow$ Pré & Pré & Pós & Dif. Pós $\rightarrow$ Pré & Pré & Pós & Dif. Pós $\rightarrow$ Pré \\
\hline Mínimo de acertos & 4 & 2 & $-50,0 \%$ & 4 & 4 & $0,0 \%$ & 4 & 8 & $100,0 \%$ \\
\hline Máximo de acertos & 10 & 10 & $0,0 \%$ & 10 & 10 & $0,0 \%$ & 10 & 10 & $0,0 \%$ \\
\hline Média de acertos & 7,4 & 7,5 & $1,7 \%$ & 7,5 & 8,5 & $13,4 \%$ & 8,3 & 9,2 & $10,6 \%$ \\
\hline Maior ou igual a 9,0 & 15 & 21 & $40,0 \%$ & 4 & 7 & $75,0 \%$ & 14 & 21 & $50,0 \%$ \\
\hline Maior ou igual a 7e menor que 8,9 & 19 & 11 & $-42,1 \%$ & 3 & 2 & $-33,3 \%$ & 8 & 5 & $-37,5 \%$ \\
\hline Maior ou igual a 5 e menor que 6,9 & 13 & 14 & $7,7 \%$ & 3 & 1 & $-66,7 \%$ & 3 & 0 & $-100,0 \%$ \\
\hline Menor do que 5 & 2 & 3 & $-33,3 \%$ & 1 & 1 & $0,0 \%$ & 1 & 0 & $-100,0 \%$ \\
\hline
\end{tabular}

Figura 3: Diferença de conhecimento entre pré-teste e pós-teste.

\subsection{Analise dos registros digitais}

Buscando melhor entender se e como as escalas de Flow estão relacionadas com um melhor desempenho acadêmico, foi aplicada a técnica de clustering KMeans nos indicadores coletados nos questionários e no registro digital em banco de dados. Primeiramente foram agrupados os dados da escala de Flow e da nota do Pós-Teste, apresentado na Figura 4 na tabela "1) Flow X Pós-Teste", onde pode-se verificar o crescimento no Flow percebido e na nota do Pós-Teste do cluster1 para o cluster2 e do cluster2 para o cluster3, indicando que os alunos com maior escala de Flow obtiveram as melhoras notas no Pós-Teste. Um resultado um pouco diferente foi encontrado na clusterização da escala de Flow e da pontuação nos Quizzes, vista na Figura 4 na tabela "2) Flow X Quizzes", onde o cluster1 apresenta a maior escala de Flow, porém a menor pontuação nos Quizzes, o cluster2 mostra a escala de Flow e a pontuação nos Quizzes intermediária, enquanto o cluster3 exibe uma escala de Flow muito próxima do cluster1, junto com a maior pontuação nos Quizzes. Acredita-se que o cluster3 demonstra que maiores escalas de Flow podem levar aos melhores resultados acadêmicos.

Outra clusterização envolveu a escala de Flow, a pontuação nos Quizzes e a nota do Pós-Teste, demonstrada na Figura 4 na tabela “3) Flow X Quizzes X Pós-Teste”. Este agrupamento mostra que o crescimento da escala de Flow foi acompanhada por 
maiores pontuações nos Quizzes e por maiores notas no Pós-Teste, de forma semelhante aos resultados da tabela "1) Flow X Pós-Teste". Por fim, a clusterização que envolveu a escala de Flow, a pontuação nos Quizzes, a nota do Pós-Teste e os pontos nas tarefas realizadas, visualizado na Figura 4, na tabela "4) Flow X Quizzes X Pós-Teste X Pontos", também exibe incremento na escala de Flow juntamente com os indicadores: a pontuação no Quizzes, a nota do Pós-Teste e os pontos nas tarefas realizadas.

\begin{tabular}{|c|c|c|c|c|c|c|c|c|c|c|c|c|c|c|}
\hline \multicolumn{3}{|c|}{ Relação Flow X Pos Teste } & \multicolumn{3}{|c|}{ Relação Flow X Quizzes } & \multicolumn{4}{|c|}{ Relação Flow X Quizzes X Pós-Teste } & \multicolumn{5}{|c|}{ Relação Flow X Quizzes X Pós-Teste X Pontos } \\
\hline \multicolumn{3}{|c|}{ Cluster 1} & \multicolumn{3}{|c|}{ Cluster 1} & \multicolumn{4}{|c|}{ Cluster 1} & \multicolumn{5}{|c|}{ Cluster 1} \\
\hline \multirow{4}{*}{$\begin{array}{l}\text { Minimo } \\
\text { Máximo } \\
\text { Média }\end{array}$} & FLOW & Pós & \multirow{4}{*}{$\begin{array}{l}\text { Minimo } \\
\text { Máximo } \\
\text { Média }\end{array}$} & FLOW & Quizzes & \multirow{4}{*}{$\begin{array}{l}\text { Minimo } \\
\text { Máximo } \\
\text { Média }\end{array}$} & FLOW & \multirow{2}{*}{$\begin{array}{c}\text { Quizzes } \\
1,00\end{array}$} & \multirow{2}{*}{$\begin{array}{l}\text { Pós } \\
2,00\end{array}$} & \multirow{3}{*}{$\begin{array}{l}\text { Minimo } \\
\text { Máximo }\end{array}$} & FLOW & Quizzes & Pós & Pontos \\
\hline & 2,50 & 2,00 & & 2,50 & 1,00 & & 2,50 & & & & 2,50 & 1,00 & 2,00 & 0,40 \\
\hline & 4,69 & 7,00 & & 4,69 & 3,53 & & 4,69 & 6,43 & 7,00 & & 4,69 & 5,92 & 7,00 & 3,00 \\
\hline & 3,58 & 5,61 & & 3,78 & 2,21 & & 3,60 & 2,98 & 5,28 & Média & 3,62 & 2,77 & 5,14 & 1,45 \\
\hline \multicolumn{3}{|c|}{ Cluster 2} & \multicolumn{3}{|c|}{ Cluster 2} & \multicolumn{4}{|c|}{ Cluster 2} & \multicolumn{5}{|c|}{ Cluster 2} \\
\hline \multirow{3}{*}{$\begin{array}{l}\text { Minimo } \\
\text { Máximo }\end{array}$} & FLOW & Pós & \multirow{4}{*}{$\begin{array}{l}\text { Minimo } \\
\text { Máximo } \\
\text { Média }\end{array}$} & FLOW & Quizzes & \multirow{4}{*}{$\begin{array}{l}\text { Minimo } \\
\text { Máximo } \\
\text { Média }\end{array}$} & FLOW & Quizzes & Pós & \multirow{4}{*}{$\begin{array}{l}\text { Minimo } \\
\text { Máximo } \\
\text { Média }\end{array}$} & FLOW & Quizzes & Pós & Pontos \\
\hline & 2,86 & 8,00 & & 2,67 & 4,07 & & 2,86 & 2,47 & 6,00 & & 2,86 & 2,47 & 5,00 & 0,80 \\
\hline & 4,75 & 9,00 & & 4,47 & 6,03 & & 4,47 & 6,42 & 10,00 & & 4,47 & 6,43 & 10,00 & 3,70 \\
\hline Média & 3,78 & 8,67 & & 3,62 & 4,91 & & 3,76 & 4,90 & 8,57 & & 3,63 & 4,36 & 7,71 & 1,95 \\
\hline \multicolumn{3}{|c|}{ Cluster 3} & \multicolumn{3}{|c|}{ Cluster 3} & \multicolumn{4}{|c|}{ Cluster 3} & \multicolumn{5}{|c|}{ Cluster 3} \\
\hline \multirow{4}{*}{$\begin{array}{l}\text { Minimo } \\
\text { Máximo } \\
\text { Média }\end{array}$} & FLOW & Pós & \multirow{4}{*}{$\begin{array}{l}\text { Minimo } \\
\text { Máximo } \\
\text { Média }\end{array}$} & FLOW & Quizzes & \multirow{4}{*}{$\begin{array}{l}\text { Minimo } \\
\text { Máximo } \\
\text { Média }\end{array}$} & FLOW & Quizzes & Pós & \multirow{4}{*}{$\begin{array}{l}\text { Minimo } \\
\text { Máximo } \\
\text { Média }\end{array}$} & FLOW & Quizzes & Pós & Pontos \\
\hline & 3,39 & 10,00 & & 2,92 & 6,22 & & 3,11 & 6,22 & 7,00 & & 3,11 & 4,43 & 6,00 & 1,30 \\
\hline & 4,47 & 10,00 & & 4,75 & 9,00 & & 4,75 & 9,00 & 10,00 & & 4,75 & 9,00 & 10,00 & 4,10 \\
\hline & 3,94 & 10,00 & & 3,77 & 7,29 & & 3,82 & 7,39 & 9,06 & & 3,85 & 7,05 & 9,00 & 2,50 \\
\hline
\end{tabular}

Figura 4: Clusters de Flow, Pós-Teste, Quizzes, Pontos e suas combinações.

\section{Discussão dos Resultados}

Com base na análise dos dados percebeu-se que a utilização da metodologia ARCS para o desenvolvimento do Laboratório Educacional Virtual proporcionou os elementos de design que tornaram as atividades propostas atrativas para os alunos. Mais, a ordem em que a estruturação das atividades foram disponibilizadas no LEV: (I) materiais educacionais (AUSUBEL, 1978; MAYER, 2001; MORENO e MAYER, 2007, SWELLER, 2005), (II) experimentos práticos (KOLB, 1984; BLOOM, 1986; MYLLER et al. 2009; KELLER, 2000; ZICHERMANN e CUNNINGHAM, 2011) e (III) testes de conhecimento (Quizzes) (KOLB, 1984; BLOOM, 1986), oportunizaram o engajamento do aluno e consequentemente, possibilitaram vivenciar o estado de Flow.

A compilação das respostas dos alunos ao Questionário de Flow (Flow State Scale-2) permitiu identificar que o laboratório educacional virtual criou condições para que os alunos vivenciassem o estado de Flow durante a execução das atividades práticas propostas nos laboratórios (JACKSON; EKLUND e MARTIN, 2010). Também, observou-se que a detecção dos indícios da presença de Flow durante as práticas no laboratório educacional virtual, não foi possível de ser detectada diretamente, uma vez que o estado de Flow é um estado interno do indivíduo (CSIKSZENTMIHALYI, 1990; WORSLEY e BLIKSTEIN, 2015) e o mundo virtual OpenSim, na forma de distribuição escolhida, não possui recursos para este fim.

Considerando as escalas das dimensões de Flow apresentadas na Figura 4, pode-se crer que os alunos do grupo GA11 permaneceram mais engajados no LEV, uma vez que percorreram todo o trajeto do ambiente virtual, realizaram as atividades, em sua maioria, e preencheram todos os testes eletrônicos. Também, identifica-se que os alunos com a maior percepção de Flow (grupos GA11 e POS8) obtiveram melhores desempenhos no Pós-Teste.

Por fim, verificou-se que a diferença de acertos entre o Pós-Teste e Pré-Teste (Figura 3), identifica um considerável crescimento na média destes acertos dos grupos GA11 e POS8 em relação ao Geral; da mesma forma, percebe-se que as notas iguais ou maiores a 9,0 do Pós-Teste aumentaram para os alunos no Geral, mas, foram ainda maiores para o GA11 e para o POS8. Ainda, as clusterizações presentes na Figura 4 mostram que o crescimento de Flow é acompanhado por um melhor desempenho acadêmico, tanto nos testes eletrônicos quanto no Pós-Teste. 


\subsection{Limitações}

Uma limitação presente nesta pesquisa é a impossibilidade de identificação da existência do estado de Flow no ambiente virtual de forma direta. Esta limitação é justificada pela concepção do projeto desta pesquisa, onde foram observadas as limitações tecnológicas existentes nas escolas públicas, assim, não foram utilizados dispositivos e/ou sensores para monitorar os sinais fisiológicos e comportamentais dos alunos, o que traria indicativos mais precisos do estado de Flow, mas não poderia ser aplicado facilmente nas escolas públicas.

\section{Conclusões}

Em decorrência da realização desta pesquisa, foi possível perceber a importância de criar um ambiente atrativo e engajador, com todas as atividades organizadas e estruturadas, possuindo informações claras sobre sua realização e com feedback imediato do resultado de sua execução, de modo que motive o aluno para $o$ cumprimento de todas as etapas teórico-práticas propostas no ambiente, de tal forma que o estudante sinta-se profundamente concentrado, envolvido e satisfeito, a ponto de se perceber no estado de Flow, por que esta condição promove uma melhor aprendizagem, e portanto melhores resultados.

Então, como grande contribuição para a ciência, para os estudos de Flow e para a melhoria do processo de ensino e aprendizagem, apresentou-se um estudo que permitiu elicitar fatores que impactam ou não no estado de Flow, e se um estado de Flow mais elevado realmente resulta em uma melhor aprendizagem ou não; mostrando, apesar das correlações positiva um tanto baixas, que o comportamento dos alunos é bastante variado e que há, como em todos os ambientes, exceções, e apesar do mundo virtual ser o mesmo, alguns estudantes se declararam em elevado estado de Flow, sem ter realizado as atividades, que do ponto de vista de aprendizagem, eram importantes.

Por fim, pode-se concluir que a vivência do estado de Flow proporciona circunstâncias que levam a uma melhor aprendizagem e um melhor desempenho acadêmico. Mas, nem sempre todos os alunos que se perceberam no estado de Flow, alcançaram bons resultados no Pós-Teste, o que levanta a conclusão de que o estado de Flow por si só é o elemento catalisar, mas não definitivo para a melhor aprendizagem.

\subsection{Trabalhos Futuros}

Um trabalho futuro para esta pesquisa é a ampliação dos conteúdos abordados, bem como a disponibilização de outras áreas de ciências e matemática. Uma lacuna existente neste trabalho é a detecção dos indícios do estado de Flow durante a execução das atividades práticas no laboratório educacional virtual. Uma possibilidade de extensão desta pesquisa é a utilização de sensores psicométricos e/ou biométricos conectados ao aluno (no mundo físico) e sua respectiva relação com as ações do avatar (no mundo virtual), formando um circuito fechado de retroalimentação das ações no mundo virtual e dos estados de humor do aluno, conforme os trabalhos de (WORSLEY et al., 2015; KEROUS et al., 2018 e LABONTE-LEMOYNE et al., 2018).

\section{Referências}

Auer, M.; Pester, A.; Ursutiu, D. e Samoila, C. Distributed Virtual and Remote Labs in Engineering. In: International Conference On Industrial Technology.2003 N.2,p.1208-1213. Ausubel, D. P.; Novak J. D. e Hanesian, H. Educational Psychology: A Cognitive View. In New York: Holt, Rinehart\& Winston, 1978.

Busarello, R.I.; Ulbricht, V.R. e Fadel, L.M. A gamificação e a sistemática de jogo: conceitos sobre a gamificação. In: Gamificação na educação. São Paulo: Pimenta Cultural, 2014. 300p. 
CETIC.BR. Pesquisa sobre o uso das tecnologias da informação e comunicação nas escolas brasileiras. São Paulo: CGIBr, 2017. Disponível: <http://cetic.br/publicacao/pesquisa-sobre-ouso-das-tecnologias-de-informacao-e-comunicacao-nas-escolas-brasileiras-tic-educacao-2016/>. Csikszentmihalyi, M. Flow: The Psychology of Optimal Experience, HarperCollins, 1990. e-Tech. CloudLabs | e-Tech CloudLabs - Laboratorios Virtuales. 2017. Disponível em: $<$ http://www.etechcloudlabs.com/index.php/en/home>. Acesso em Mai. 2018.

Feisel, L. D. e Rosa, A. J. The Role of the Laboratory in Undergraduate Engineering Education, In: Journal of Engineering Education.Vol. 94, N. 1, 2005, pp. 121-130.

GAMIFICATION.ORG. Welcome to the Gamification.org! | Gamification Wiki. 2010. Disponível em: https://badgeville.com/wiki/. Acesso em 25 Abr. 2015.

HARVARD. ecoMUVE Overview. 2011. Disponível em: < https://ecolearn.gse.harvard. edu/ecoMUVE/overview.php>.

Jackson, S.; Eklund, B. e Martin, A. The FLOW Manual: The Manual for the Flow Scales. 2010. Disponível em: <https://www.mindgarden.com/100-flow-scales>.

Kapp, K. M. The Gamification of Learning and Instruction: game-based methods and strategies for training and education. Pfeifer, Wiley USA, 2012.

Keller, J. M. Motivational design for learning and performance: the ARCS model approach. New York, Springer. 2010. Disponível em: http://public.eblib.com/choice/publicfullrecord.aspx?p=510896. Acesso em: 07 Set. 2016.

Kerous, B; Skola, F. e Liarokapis, F. EEG-based BCI and video games: a progress report. Virtual Reality, Vol. 22, Núm. 2, pág.119-135, 2018.

Kolb, D. Experiential Learning: Experience as the Source of Learning and Development, Prentice Hall, Englewood Clis, 1984.

Labonte-LeMoyne et al. Are We in Flow Neurophysiological Correlates of Flow States in a Collaborative Game. In: Proceedings of Conference Extended Abstracts on Human Factors in Computing Systems, pg. 1980-1988. 2016.

Labster. Labster Virtual Lab Simulations. Disponível em: <https://www.labster.com/>.

Mayer, R. E. ; Heiser, J. e Lonn, S. Cognitive constraints on multimedia learning. Journal of Educational Psychology, V. 93, N. 1, pag. 187-198. 2001.

Moreno, R. e Mayer, R. Interactive Multimodal Learning Environments - Special Issue on Interactive Learning Environments: Contemporary Issues and Trends. Educational Psychology Review. Vol. 19, Ed. 3, pág. 309-326, 2007.

Nelson, B.C.; Erlandson, B.E. Design for Learning in Virtual Worlds: Interdisciplinary Approaches Educational Technology. New York: Routledge. 2012.

NMC e FIRJAN. Perspectivas tecnológicas para o ensino fundamental e Médio Brasileiro de 2012 a 2017, 2012. Disponível em: <http://zerohora.clicrbs.com.br/pdf/14441735.pdf>.

Occhioni, M. Techland, a virtual world for maths and science, In: Proceedings of the 3rd European Immersive Education Summit, 2013, pp. 94-99.

Piaget, J. The Principles of Genetic Epistemology, Routledge \& Kegan, London: 1972.

Santaella, L. Cultura e artes do pós-humano. São Paulo: Paulus. 2003.

Schäfer, C. e Lopes, T.R.C. Cultura do software e autonomização da game music. In: Anais V

Simpósio Nacional ABCiber, 2012. Disponível em: <http://www.abciber .org/simposio2011/anais/Trabalhos/artigos/Eixo\%204/5.E4/162.pdf>.

Sciethics Interactive. 2013. Disponível em: $<$ http://www.sciethicsinteractive.com/>.

Sweller J. e Merrienboer J. Cognitive Load Theory and Complex Learning: Recent Developments and Future Directions. Educational Psychology Review, V.17, N.2, 2005.

Tibola, L.R. e Tarouco, L.M.R. Rastreamento de Interações em Laboratórios Educacionais nos Mundos Virtuais 3D para Identificação de Engajamento. In: RENOTE, v.13, n.2, 2015.

Worsley M. e Blikstein P. Leveraging multimodal learning analytics to differentiate student learning strategies. In: Proceedings of the Fifth International Conference on Learning Analytics And Knowledge, pages 360-367. ACM, 2015.

Worsley, M.; Scherer, S.; Blikstein, P. e Morency, L-P. Exploring Behavior Representation for Learning Analytics. In: Proceeding of the 2015 ACM on International Conference on Multimodal Interaction, ACM, Pág. 251-258, 2015. 